\title{
Acumulación de biomasa en yuca (Manihot esculenta C.) ante la respuesta a la aplicación de fertilizantes NPK y biofertilizantes en condiciones de invernadero en dos tipos de suelo
}

\section{Biomass accumulation response of cassava (Manihot esculenta C.) to NPK fertilization and biofertilizers in two soil types under greenhouse conditions}

JAZMÍN VANESSA PÉREZ-PAZOS'1

LILY LORENA LUNA-CASTELLANOS ${ }^{1}$ OSCAR BURBANO-FIGUEROA ${ }^{1,2}$

Cultivo de yuca, establecido en casa malla. Foto: J.V. Pérez-Pazos

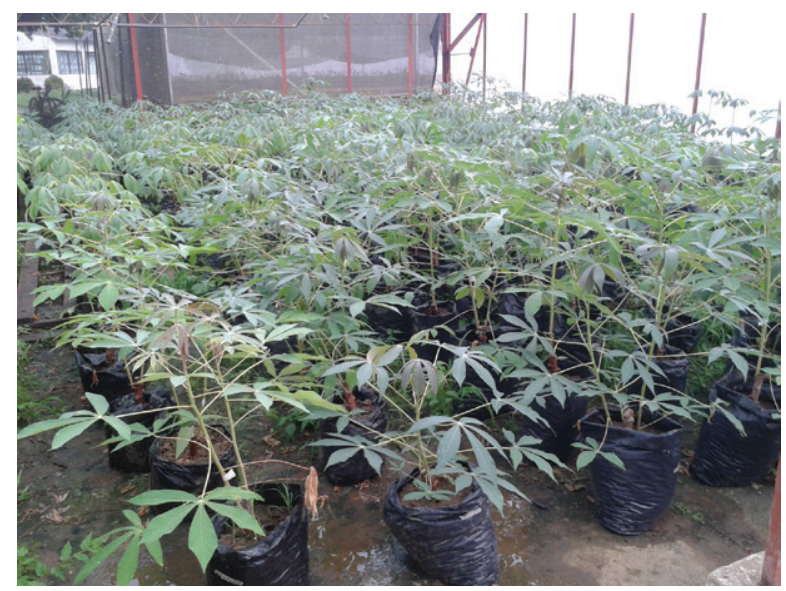

\section{RESUMEN}

El cultivo de la yuca en el Caribe colombiano es un sistema productivo con un bajo nivel de integración de tecnologías modernas sustentables que permitan incrementar la rentabilidad del cultivo. La validación de prácticas asociadas a la fertilización conforma una de las alternativas para incluir en las estrategias de manejo integrado del cultivo. El objetivo de este estudio fue evaluar a nivel de casa malla, la respuesta de acumulación de biomasa en plantas de yuca a la fertilización NPK en dos tipos de suelos (arenoso y franco-arcilloso) y en presencia de tres biofertilizantes. El efecto de diferentes inoculantes microbianos comercialmente disponibles, Azobac $^{\circledR}$ (Azotobacter chrococcum), Fosforiz ${ }^{\circledR}$ (Pseudomonas fluorescens) y micorrizas arbusculares (Safer Micorrizas $\left.^{\circledR}\right)$, fue evaluado en mezcla con diferentes niveles de fertilización inorgánica (100, 75, 50, 25 y 0\%), más los testigos sin inoculación en un arreglo factorial $2 \times 3 \times 5$. Los resultados obtenidos indican que el nivel de fertilización NPK en el que se alcanza la mayor acumulación relativa de biomasa ocurre al 25\% de la mezcla. Los mayores valores de acumulación de biomasa se alcanzan con la presencia de micorrizas. Azobac ${ }^{\circledR}$ y Fosforiz $^{\circledR}$ no exhibieron ningún efecto sobre la acumulación de biomasa. Los resultados obtenidos sugieren que los biofertilizantes comerciales basados en micorrizas pueden ser usados para maximizar la respuesta a la fertilización con NPK.

\footnotetext{
The Plant Interactions Laboratory, Centro de Investigación Turipaná, Corporación Colombiana de Investigación Agropecuaria (Corpoica, ahora Agrosavia), Cereté (Colombia). ORCID Pérez-Pazos, J.: 0000-0002-1889-8248; ORCID Luna-Castellanos, L.: 0000-0003-2172-7842; ORCID Burbano-Figueroa, O.: 0000-0002-6604-7333

2 Autor para correspondencia. burbano.figueroa1@gmail.com
} 


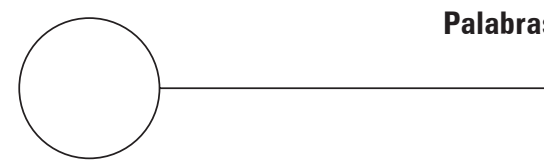

Palabras clave adicionales: nutrición vegetal, bacterias promotoras de crecimiento, hortalizas de raíz, cultivos de almidón, región Caribe colombiana, suelo arenoso, suelo franco arcilloso.

\section{ABSTRACT}

The adoption of sustainable technologies that are able to increase profitability in the cassava production systems of the Colombian Caribbean is very limited. The assessment of alternative fertilization practices is required for the development of integrated approaches for crop management. The objective of this research was to estimate how the biomass accumulation response of cassava plants to NPK fertilization is affected by the inoculation of three biofertilizers under greenhouse conditions in two soil types (sandy and clayey loam soils). The selected biofertilizers are commercial products available in the Colombian market: Azobac ${ }^{\circledR}$ (Azotobacter chrococcum), Fosforiz ${ }^{\circledR}$ (Pseudomonas fluorescens) and arbuscular mycorrhizal fungi (Safer Micorrizas ${ }^{\circledR}$ ). NPK was applied at varying levels of the recommended nutrients requirements $(100,75,50,25$ and $0 \%)$ in inoculated and non-inoculated (control) treatments in a $2 \times 3 \times 5$ factorial design. The highest biomass accumulation was obtained at the lower fertilization levels (25\%) in the treatment inoculated with arbuscular mycorrhizal fungi. The cassava plants inoculated with Azobac ${ }^{\circledR}$ and Fosforiz $z^{\circledast}$ did not exhibit increased biomass accumulation. Our results suggest that commercial biofertilizers based on mycorrhizal fungi can be used for maximizing cassava responses to NPK fertilization.

Additional key words: plant nutrition, growth promoting bacteria, root vegetables, starch crops, Colombian Caribbean region, sandy soil, clay loam soil.

Fecha de recepción: 25-01-2018 Aprobado para publicación: 30-05-2018

INTRODUCCIÓN

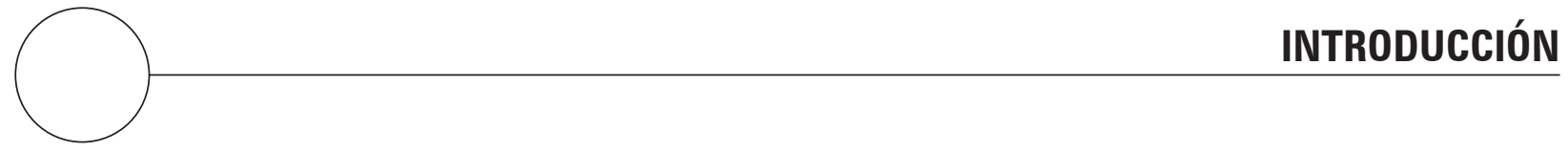

La yuca es uno de los productos agrícolas de importancia para la seguridad alimentaria de la mayoría de los países en vías de desarrollo (DANE, 2004; Vila, 2012). En el 2016 a nivel mundial, se registró un área cultivada de 23,5 millones de hectáreas (FAO, 2016), de las cuales la mitad corresponde a los países en vía de desarrollo, donde la facilidad del cultivo y el gran contenido de energía de la yuca se ha convertido en un valioso producto comercial para millones de agricultores (João et al., 2016). En Latinoamérica, después de Brasil y Paraguay, Colombia es el tercer productor de yuca, con un área cultivada de 210.250 ha, producción de 2,1 millones de toneladas y un rendimiento promedio de 10,01 $\mathrm{t} \mathrm{ha}^{-1}$ (FAO, 2016). En la región Caribe, se concentra el $50 \%$ de la producción nacional pero no obstante, los rendimientos son bajos, como consecuencia de la deficiente nivel de integración de tecnologías (MADR, 2006). Es un producto de economía campesina con prácticas tradicionales en su manejo, poco intensivo en el uso de maquinaria agrícola, uso de semillas de calidad, fertilización de suelos e insumos químicos para el control de enfermedades y malezas (Aguilera, 2012), que permitan maximizar la productividad de las plantas.

Si bien es un cultivo rústico, los altos rendimientos del cultivo de yuca requieren de la aplicación de fertilizantes minerales o abonos orgánicos. Dosis óptimas de fertilización permiten alcanzar entre 40 y 60 t ha ${ }^{-1}$ de raíces comerciales (Howeler, 2014). Sin embargo, el uso excesivo de fertilizantes edáficos causa graves daños al medio ambiente e incrementa los costos de producción en un cultivo de subsistencia como la yuca. Una de las alternativas promisorias para la disminución del uso de los fertilizantes de tipo mineral lo constituyen los biofertilizantes (Grageda et al., 2012). Estos productos están elaborados a base de microorganismos, los cuales una vez incorporados al suelo pueden establecer relaciones generalmente de tipo benéfico con las plantas favoreciendo positivamente la nutrición, crecimiento y protección (Vessey, 2003). En el suelo existe una diversidad de microorganismos benéficos distribuidos en diferentes grupos. El primero, lo constituyen las bacterias 
promotoras del crecimiento vegetal (BPCV), definidas así por la capacidad de favorecer el crecimiento vegetal a través de mecanismos como la fijación de nitrógeno, solubilización de minerales, producción de fitohormonas, sideróforos y compuestos antagónicos a los fitopatógenos (Lugtenberg y Kamilova, 2009). En el segundo grupo se encuentran los hongos vesiculo-arbusculares (micorrizas), especialmente las endomicorrizas, las cuales se incorporan al sistema radicular de la planta favoreciendo la nutrición a través de la absorción, regulación de minerales y agua, contribuyendo a la disminución de la erosión en el suelo (Sánchez, 1999; Sylvia et al., 1999). La incorporación de micorrizas en los sistemas de cultivo incrementa la adaptación del material vegetal al entorno, generando una mayor competitividad y productividad respecto a plantas no micorrizadas (Molina et al., 2005). Con el fin de generar una línea base para el desarrollo y posterior vinculación de nuevas tecnologías asociadas al componente de fertilización dentro del modelo integrado de producción de yuca, este trabajo pretende evaluar la respuesta de acumulación de biomasa en estas plantas a la fertilización NPK en dos tipos de suelos (arenoso-arcilloso) y presencia de tres biofertilizantes.

\section{MATERIALES Y MÉTODOS}

La presente investigación se llevó a cabo durante el segundo semestre de 2013, en el Centro de Investigación (CI) Turipaná - Corpoica (ahora Agrosavia), ubicado en el km 13 vía Cereté-Montería (Córdoba, Colombia), con coordenadas geográficas 8³1'16" N y $75^{\circ} 58,11$ " W. El experimento fue desarrollado bajo condiciones controladas en invernadero, a una temperatura promedio de $30 \pm 2^{\circ} \mathrm{C}$ y humedad relativa de $80 \pm 5 \%$.

\section{Material vegetal}

Estacas de yuca de la variedad MCol-2215 o 'Venezolana' de $20 \mathrm{~cm}$ de longitud con 5-6 yemas, de buen grosor y vigor, fueron obtenidas de plantas maduras de 10 meses de edad, sanas, uniformes en diámetro y provenientes del tercio medio. Las estacas fueron desinfectadas empleando una mezcla a base de fungicida e insecticida.

\section{Suelos}

Dos tipos de suelos provenientes del Valle del Sinú (Cereté, Córdoba) y sabanas colinadas (Ciénaga de Oro, Córdoba) en la región del Caribe Colombiano fueron usados. El suelo homogeneizado se depositó en bolsas de polietileno calibre 4 de $25 \times 45 \mathrm{~cm}$, con capacidad para $4 \mathrm{~kg}$, las cuales se ubicaron en estibas plásticas. Una muestra de $1 \mathrm{~kg}$ de suelo, compuesta de 5 sub-muestras en forma de equis fue analizada para los parámetros: textura, $\mathrm{pH}$, materia orgánica, fósforo disponible y potasio intercambiable.

\section{Biofertilizantes y fertilización inorgánica}

Se emplearon los inoculantes microbianos disponibles comercialmente Azobac ${ }^{\circledR}$, Fosforiz ${ }^{\circledR}$ y Safer Micorrizas M.A. ${ }^{\oplus}$, de manera individual y en mezcla con diferentes niveles de fertilización inorgánica: 100, 75, 50,25 y $0 \%$, además de un control con los niveles de fertilización inorgánica sin inoculación.

La fertilización inorgánica se realizó con los macronutrientes nitrógeno, fósforo y potasio (NPK) aplicados en forma de urea, fosfato diamónico (DAP) y cloruro de potasio (KCl). Las dosis de cada fertilizante se calcularon teniendo en cuenta los requerimientos del cultivo de yuca por Cadavid (2002) (Tab. 1).

Tabla 1. Dosis de fertilizantes inorgánicos NPK estimados para cultivo de yuca en el Caribe colombiano.

\begin{tabular}{|l|c|c|c|c|c|c|}
\hline \multirow{2}{*}{ Requerimientos } & \multicolumn{3}{c|}{ Suelo Ciénaga de Oro } & \multicolumn{4}{c|}{ Suelo C.I. Turipaná } \\
\cline { 2 - 8 } & $\mathrm{N}$ & $\mathrm{P}_{2} \mathrm{O}_{5}$ & $\mathrm{~K}_{2} \mathrm{O}$ & $\mathrm{N}$ & $\mathrm{P}_{2} \mathrm{O}_{5}$ & $\mathrm{~K}_{2} \mathrm{O}$ \\
\hline Requerimiento elemento por cultivo $\left(\mathrm{kg} \mathrm{ha}^{-1}\right.$ ) & 120 & 50 & 100 & 120 & 25 & 80 \\
\hline Requerimiento producto (kg ha-1) & 260,9 & 108,7 & 166,7 & 260,9 & 54,3 & 133,3 \\
\hline Requerimiento producto (g/planta) Dosis 100\% & 26,1 & 10,9 & 16,7 & 26,1 & 5,4 & 13,3 \\
\hline Requerimiento producto (g/planta) Dosis 75\% & 19,6 & 8,2 & 12,6 & 19,6 & 4,1 & 10 \\
\hline Requerimiento producto (g/planta) Dosis 50\% & 13 & 5,4 & 8,4 & 13 & 2,7 & 6,6 \\
\hline Requerimiento producto (g/planta) Dosis 25\% & 6,6 & 2,7 & 4,2 & 6,6 & 1,4 & 3,4 \\
\hline
\end{tabular}


Tabla 2. Biofertilizantes incluidos en la fertilización inorgánica de yuca en el Caribe colombiano.

\begin{tabular}{|l|l|c|c|c|}
\hline \multicolumn{1}{|c|}{ Biofertilizante } & \multicolumn{1}{|c|}{ Microorganismo } & $\begin{array}{c}\text { Registro de } \\
\text { venta ICA }\end{array}$ & \multicolumn{1}{c|}{ Distribuidor } & Composición \\
\hline Azobac $^{\circledR}$ & Azotobacter chroococcum & 6520 & \multirow{2}{*}{$\begin{array}{c}\text { Fundases, Bogotá } \\
\text { (Colombia) }\end{array}$} & $14 \cdot 10^{8} \mathrm{UFC} / \mathrm{mL}$ \\
\cline { 1 - 3 } Fosforiz $^{\circledR}$ & Pseudomonas fluorescens & 6519 & Agrobiológicos & $10^{8} \mathrm{UFC} / \mathrm{mL}$ \\
\hline Safer Micorrizas M.A. ${ }^{\circledR}$ & $\begin{array}{l}\text { Glomus fasciculatum, Scutellospora heterogama, } \\
\text { Glomus mosseae, Glomus manihotis, Acaulospora rugosa } \\
\text { y Entrophospora colombiana }\end{array}$ & 7969 & $\begin{array}{c}\text { Safer, Medellín } \\
\text { (Colombia) }\end{array}$ & 240 esporas/g \\
\hline
\end{tabular}

El fósforo se aplicó al momento de la siembra mientras que el nitrógeno y el potasio fueron aplicados 30 días después de la siembra (dds).

La aplicación de biofertilizantes se realizó con los productos descritos en la tabla 2. Las micorrizas arbusculares se aplicaron al momento de la siembra en una dosis de $40 \mathrm{~g} /$ planta. Azobac ${ }^{\circledR}$ y Fosforiz ${ }^{\circledR}$ fueron agregados 38 dds en las dosis recomendadas por el fabricante en dosis de 3 y $5 \mathrm{~L} \mathrm{ha}^{-1}$, respectivamente.

\section{Diseño experimental}

El experimento se realizó bajo un diseño de bloques completamente aleatorizados con un arreglo factorial $2 \times 3 \times 5$, con tres repeticiones, dos tipos de suelo (Cienaga de Oro-CIO y Turipaná-TUR), tres biofertilizantes (Azobac-AZO; Fosforiz-FOS y Micorrizas-MIC) y cinco niveles de fertilización (0, 25, 50, 75 y 100\%). La unidad experimental (UE) en este estudio estuvo conformada por grupos de 10 plantas. Como variable respuesta, se determinó el peso fresco y seco de las raíces tuberosas a los 120 dds, en cada uno de los tratamientos. Se realizó un análisis de varianza y el Test de Fisher para comparación de medias. La correlación entre los datos de peso fresco y seco fue evaluada con el propósito de disminuir auto correlaciones en el análisis final. El peso fresco fue usado para determinar la acumulación relativa de biomasa (rBioAcc). Para todos los análisis y generación de gráficos se empleó el software estadístico Infostat (Rienzo et al., 2015).

\section{RESULTADOS Y DISCUSIÓN}

El análisis para cada uno de los suelos indica que el suelo de Ciénaga de Oro (CIO) presentó textura arenosa, $\mathrm{pH}$ extremadamente ácido de 4,5, bajo contenido de materia orgánica $(0,58 \%)$ y minerales representados como fósforo disponible (P) $\left(1,2 \mathrm{mg} \mathrm{kg}^{-1}\right)$ y potasio intercambiable $(\mathrm{K})\left(0,06 \mathrm{cmol}_{(+)} \mathrm{kg}^{-1}\right)$, en general este suelo tiene una baja calidad nutricional (Tab. 3). En contraste, el suelo de Turipaná (TUR) aunque presenta niveles bajos de $\mathrm{P}\left(25,44 \mathrm{mg} \mathrm{kg}^{-1}\right)$ y $\mathrm{K}\left(0,67 \mathrm{cmol}_{(+)}\right.$ $\left.\mathrm{kg}^{-1}\right)$, los valores superan significativamente a los encontrados en $\mathrm{CIO}$, el contenido de materia orgánica es superior $(2,57 \%)$, el $\mathrm{pH}$ se considera como normal $(6,55)$ y la textura es franco-arcilloso, estas características hacen de este tipo de suelo favorable para el establecimiento de sistemas productivos de yuca (Suárez y Mederos, 2011).

Se encontraron efectos significativos del tipo de suelo (SLOC), biofertilizantes (BIOF) y niveles de NPK sobre la acumulación relativa de biomasa (rBioAcc). Adicionalmente, se presentaron interacciones significativas entre los siguientes factores: SLOC*BIOF $(P \leq 0,01)$, SLOC*NPK $(P \leq 0,001)$, BIOF*NPK $(P \leq 0,001)$ y SLOC*BIOF*NPK $(P \leq 0,01)$ (Tab. 4$)$.

Dentro de los 5 niveles de fertilización NPK sugeridos por Cadavid (2002), la respuesta de rBioAcc exhibió 2 puntos máximos, a niveles del 25 y 100\% (Tab. 5).

Una mayor acumulación relativa de biomasa fue observada en los suelos arenosos (CIO) en comparación con los suelos arcillosos (TUR) (Tab. 3) (Fig. 1A). El mayor crecimiento relativo de las plantas de yuca en los suelos CIO en comparación con TUR puede ser explicado por el mayor efecto combinado de la fertilización NPK y BIOF en suelos más pobres (Tab. 4).

La carga de P y K (g/planta) aplicada en los suelos CIO es dos veces la aplicada a los suelos TUR en consideración a las diferencias intrínsecas en la fertilidad de los suelos evaluados. Por lo tanto, las especies de yuca se consideran muy dependientes de su hongo simbionte en condiciones ambientales menos fértiles (Carretero et al., 2009). En el suelo de CIO el mayor incremento en la acumulación de rBioAcc está motivada por el aumento en la absorción del fósforo a 
Tabla 3. Propiedades fisicoquímicas de los dos tipos de suelos incluidos en la evaluación de factores que afectan la acumulación de biomasa en cultivos de yuca en función de NPK y biofertilizantes.

\begin{tabular}{|c|c|c|c|c|c|c|}
\hline \multirow{2}{*}{ Determinación analítica } & \multirow{2}{*}{ Unidad } & \multirow{2}{*}{ Método } & \multicolumn{2}{|c|}{ Suelo Cienaga de Oro } & \multicolumn{2}{|c|}{ Suelo C.I. Turipaná } \\
\hline & & & Valor & Interpretación & Valor & Interpretación \\
\hline Textura al tacto & & Organoléptico & $A$ & Arenoso fuerte & $\mathrm{FAr}$ & Franco-arcilloso \\
\hline pH & & Potenciómetro & 4,56 & Extremadamente ácido & 6,55 & Extremadamente ácido \\
\hline Conductividad eléctrica & $d S m^{-1}$ & $\begin{array}{l}\text { Conductivimetro } \\
\text { suelo: agua } 1: 2,5\end{array}$ & 0,12 & No salino & 0,31 & No salino \\
\hline Materia orgánica (M0) & $\%$ & Walkey y Black & 0,58 & NA & 2,57 & NA \\
\hline Acidez intercambiable $(\mathrm{Al}+\mathrm{H})$ & \multirow{7}{*}{$\mathrm{cmol}_{(+)} \mathrm{kg}^{-1}$} & \multirow{2}{*}{$\mathrm{KCl}$} & 0,25 & NA & 0,00 & NA \\
\hline Aluminio intercambiable (Al) & & & 0,00 & NA & 0,00 & NA \\
\hline Calcio intercambiable (Ca) & & \multirow{4}{*}{$\begin{array}{l}\text { Acetato de amonio } \\
1 \mathrm{~N} \mathrm{pH} \mathrm{7,0}\end{array}$} & 1,05 & Bajo & 10,07 & Bajo \\
\hline Magnesio intercambiable (Mg) & & & 0,53 & Bajo & 6,72 & Bajo \\
\hline Potasio intercambiable (K) & & & 0,06 & Bajo & 0,67 & Bajo \\
\hline Sodio intercambiable (Na) & & & 0,05 & Normal & 0,12 & Normal \\
\hline $\begin{array}{l}\text { Capacidad de intercambio } \\
\text { catiónico (CICE) }\end{array}$ & & Suma de cationes & 1,94 & Bajo & 17,58 & Bajo \\
\hline Hierro disponible (Fe) Olsen & \multirow{7}{*}{$\mathrm{mg} \mathrm{kg}^{-1}$} & \multirow{4}{*}{ Olsen modificado } & 61,40 & Alto & 80,60 & Alto \\
\hline Manganeso disponible (Mn) Olsen & & & 20,80 & Alto & 4,80 & Alto \\
\hline Zinc disponible (Zn) Olsen & & & 1,80 & Medio & 1,70 & Medio \\
\hline Cobre disponible (Cu) Olsen & & & 1,80 & Medio & 7,30 & Medio \\
\hline Fosforo disponible (P) Bray II & & Bray II & 1,20 & Bajo & 25,44 & Bajo \\
\hline Azufre disponible (S) & & \multirow{2}{*}{$\begin{array}{l}\text { Fosfato monobásico } \\
\text { calcio }\end{array}$} & 3,00 & Bajo & 8,90 & Bajo \\
\hline Boro disponible (B) & & & 0,15 & Bajo & 0,32 & bajo \\
\hline
\end{tabular}

NA: no aplica.

Tabla 4. Análisis de varianza del efecto de tipo de suelos (SLOC), biofertilizantes (BIOF) y niveles de NPK sobre la acumulación relativa de biomasa (rBioAcc) en yuca.

\begin{tabular}{|l|c|c|c|c|c|}
\hline $\begin{array}{c}\text { Fuente de } \\
\text { variación }\end{array}$ & SS & df & MS & F & Valor $\boldsymbol{P}$ \\
\hline Model. & 3,8 & 39 & 0,1 & 3,69 & $<0,0001$ \\
\hline SLOC & 0,18 & 1 & 0,18 & 6,65 & 0,0112 \\
\hline BIOF & 0,4 & 3 & 0,13 & 5,11 & 0,0024 \\
\hline NPK & 0,95 & 4 & 0,24 & 8,99 & $<0,0001$ \\
\hline SLOC ${ }^{*}$ BIOF & 0,31 & 3 & 0,1 & 3,93 & 0,0104 \\
\hline SLOC*NPK & 0,57 & 4 & 0,14 & 5,45 & 0,0005 \\
\hline BIOF*NPK & 1,04 & 12 & 0,09 & 3,3 & 0,0004 \\
\hline SLOC*BIOF*NPK & 0,74 & 12 & 0,06 & 2,34 & 0,0103 \\
\hline Error & 2,93 & 111 & 0,03 & & \\
\hline Total & 6,73 & 150 & & & \\
\hline
\end{tabular}

través de hifas extra radicales de la micorriza, que permiten una absorción más eficiente de los nutrientes presentes en el suelo (Falcón, 2010), permitiendo que en condiciones de baja disponibilidad de nutrientes como las presentes en los suelos de CIO, las plantas superen esas deficiencias generando resultados óptimos. En contraste, el hongo simbionte se ve favorecido con el incremento en el metabolismo vegetal ya que utiliza los productos de la planta como exudados y otros compuestos para realizar sus principales funciones (Cuesta et al., 2007).

La evaluación del efecto de la adición de NPK sin biofertilizantes (CTRL), revela que la acumulación de biomasa es directamente proporcional a la concentración de NPK alcanzando el pico más alto con el 100\% de fertilización. Para los tratamientos con adición de biofertilizantes se observa un punto máximo de acumulación de biomasa al 25\% de fertilización NPK que está relacionado con el efecto de la interacción de estas dos variables (BIOF*NPK) (Fig. 1B). 
Tabla 5. Efecto de tipo de suelos (SLOC), biofertilizantes (BIOF) y niveles de NPK sobre la acumulación relativa de biomasa (rBioAcc) en yuca.

\begin{tabular}{|c|c|c|c|}
\hline \multirow{2}{*}{ Factor } & \multicolumn{3}{c|}{ Acumulación relativa de biomasa } \\
\cline { 2 - 4 } & Media & \multicolumn{2}{c|}{ SE } \\
\hline NPK (\%) & 0,32 & 0,02 & a \\
\hline 0 & 0,55 & 0,04 & $\mathrm{~d}$ \\
\hline 25 & 0,41 & 0,03 & $\mathrm{bc}$ \\
\hline 50 & 0,41 & 0,03 & $\mathrm{~b}$ \\
\hline 75 & 0,49 & 0,05 & $\mathrm{~cd}$ \\
\hline 100 & LSD: 0,07945 & & \\
\hline \multicolumn{4}{|c|}{} \\
\hline Tipo de suelos & 0,40 & 0,02 & $\mathrm{a}$ \\
\hline TUR & 0,47 & 0,03 & $\mathrm{~b}$ \\
\hline CIO & LSD: 0,05616 & & \\
\hline \multicolumn{4}{|c|}{} \\
\hline Biofertilizantes & 0,36 & 0,03 & $\mathrm{a}$ \\
\hline FOS & 0,39 & 0,03 & $\mathrm{a}$ \\
\hline AZO & 0,49 & 0,04 & $\mathrm{~b}$ \\
\hline MIC & 0,50 & 0,04 & $\mathrm{~b}$ \\
\hline CTRL & LSD: 0,07947 & & \\
\hline \multicolumn{4}{|c|}{} \\
\hline
\end{tabular}

Promedios con letras distintas indican diferencia significativa según el test de Fisher $(P \leq 0,05)$.

SE: error estándar; TUR: Turipaná; CIO: Ciénaga de Oro; AZO: Azobac; FOS: Fosforiz; CTRL: control; MIC: Microrrizas.
La explicación más factible para los dos picos de respuesta observados es que la fertilización provoca efectos diferenciales sobre los microorganismos que hacen parte de los biofertilizantes, niveles bajos de fertilización incrementa el efecto de los microorganismos sobre la acumulación de la biomasa, pero niveles altos de fertilización inhiben su actividad. Reportes previos describen este tipo de efecto, donde la fertilización inorgánica tiene impacto negativo sobre la actividad biológica de microorganismos empleados como biofertilizantes (Carretero et al., 2009; Okon et al., 2010; Straker et al., 2010; Heberle et al., 2015; Séry et al., 2016; Begoude et al., 2016). En consideración a las limitantes económicas y al efecto inhibidor de elevadas cantidades de fertilización, el punto máximo de respuesta en el que se tiene interés es aquél que se presente con la menor tasa de fertilización y para esta investigación se logró con el $25 \%$. En el caso de los biofertilizantes, una mayor rBioAcc fue observada para micorrizas (MIC) en comparación con los biofertilizantes basados en bacterias de vida libre (AZOFOS) (Tab. 5). El análisis de grupos homogéneos revela que no existen diferencias significativas entre el tratamiento CTRL y el inoculado con micorrizas. La interacción entre los niveles de NPK y BIOF sobre la acumulación de biomasa refleja que la combinación de NPK al 25\% y MIC es capaz de igualar el efecto del tratamiento con fertilización completa sin biofertilizantes (NPK 100\%) (Fig. 1B).
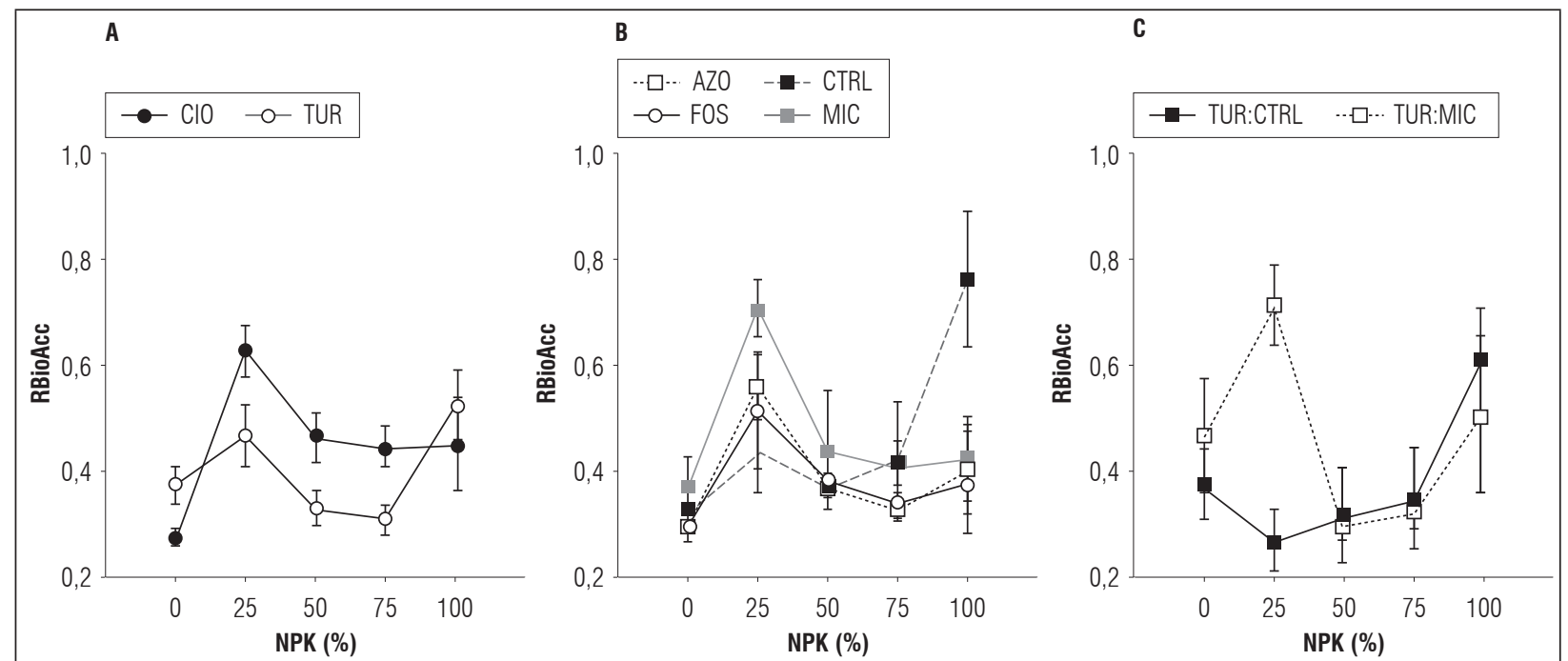

Figura 1. Efecto de concentraciones de NPK sobre la acumulación relativa de biomasa (rBioAcc) de yuca en diferentes tipos de suelos (A) y biofertilizantes (B). Interacciones entre tipos de suelos y biofertilizantes fueron observados. La interacción más fuerte se presentó entre micorrizas en el suelo de naturaleza arcillosa (C). Las barras sobre los promedios significan desviación estándar. TUR: Turipaná; CIO: Ciénaga de Oro; AZO: Azobac; FOS: Fosforiz; CTRL: control; MIC: Microrrizas. 
El cultivo de yuca es considerado micotrófico en razón de su alta dependencia de las micorrizas que tiene efecto bioprotectante y sobre el crecimiento de la planta (Okon et al., 2010; Straker et al., 2010; Heberle et al., 2015; Séry et al., 2016; Begoude et al., 2016). Esta asociación, le permite al cultivo un mayor crecimiento en la etapa inicial donde el sistema radicular es poco desarrollado (Filho y Nogueira, 2007), mejorar la eficiencia de las raíces para la extracción de nutrientes (Carretero et al., 2009; Okon et al., 2010; Séry et al., 2016) y en consecuencia, el incremento en el rendimiento del cultivo (Séry et al., 2016). Una de las especies de micorriza asociada a la yuca es Glomus manihotis, la cual es fuertemente invasiva, competitiva en el suelo y que tolera niveles altos de aplicación de fertilizante mejorando la absorción de nutrientes (Straker et al., 2010). Esta especie hace parte de los componentes de Safer Micorrizas, por lo tanto, el notorio efecto de las micorrizas en este estudio puede estar justificado por el fuerte efecto de la presencia de esta especie en el producto utilizado que tiene en las etapas iniciales del cultivo.

Como resultado de la interacción de todos los factores evaluados (SLOC*BIOF*NPK) el efecto es notoriamente visible a una concentración del $25 \%$, con el uso de micorrizas en suelos arcillosos (TUR) (Fig. 1C). Diferencias significativas fueron observadas para MIC y AZO a esta concentración (25\%) en los suelos TUR, pero ninguna tendencia fue observada en los suelos CIO. Se encuentra reportado que en plantas colonizadas por micorrizas los requerimientos nutricionales son reducidos significativamente en comparación con las no micorrizadas (Filho y Nogueira, 2007), los resultados de esta investigación indican que hay un efecto notorio de estos microorganismos en suelos arcillosos. Adicionalmente, el beneficio de la aplicación de micorrizas se encuentra en la absorción de nutrientes resultando principalmente en el aumento del área de superficie de las raíces micorrizadas e incrementando la tasa de absorción del nutriente (Filho y Nogueira, 2007). También Straker et al. (2010) encontró en suelos arcillosos como el evaluado en esta investigación, la sinergia entre micorrizas y bajos niveles de fertilización (25\%) en suelos promisorios para el desarrollo de la yuca (TUR), genera un efecto altamente significativo respecto a otras combinaciones.

\section{Conclusiones}

El efecto más significativo del uso de biofertilizantes y fertilización NPK ocurrió en los suelos más pobres de Ciénaga de Oro. La mayor respuesta de rBioAcc fue observada con el uso de micorrizas a bajas concentraciones de NPK. Este resultado sugiere que las actuales recomendaciones de fertilización están sobreestimadas y que entre los inoculantes microbianos disponibles los esfuerzos deben concentrarse en las micorrizas. Sin embargo, los resultados aquí presentados deben validarse en experimentos que incluyen rendimiento en condiciones controladas y de campo.

\section{AGRADECIMIENTOS}

Esta investigación fue desarrollada con recursos provenientes del Ministerio de Agricultura y Desarrollo Rural (CO5131 12104390011810B1) otorgados en el año 2013 y el apoyo logístico del Centro de Investigación Turipaná - Corporación Colombiana de Investigación Agropecuaria (CORPOICA). A partir de Junio del 2018, CORPOICA cambió su identidad legal a AGROSAVIA.

Conflicto de intereses: el manuscrito fue preparado y revisado con la participación de los autores, quienes declaran no tener algún conflicto de interés que coloquen en riesgo la validez de los resultados aquí presentados.

\section{REFERENCIAS BIBLIOGRÁFICAS}

Aguilera, M. 2012. La yuca en el Caribe colombiano. De cultivo ancestral a agroindustrial. Economía Regional 158. Banco de la República, Bogotá, Colombia.

Begoude, D.A.B., P.S. Sarr, T.L.Y. Mpon, D.A. Owona, M.N. Kapeua y S. Araki. 2016. Composition of arbuscular mycorrhizal fungi associated with cassava (Manihot esculenta Crantz) cultivars as influenced by chemical fertilization and tillage in Cameroon. J. Appl. Biosci. 98, 9270-9283. Doi: 10.4314/jab.v98i1.4

Cadavid, L.F. 2002. Suelo y fertilización para la yuca. Serie la yuca en el tercer milenio. CIAT, Cali, Colombia.

Carretero, C.L., M. Cantos, J.L. García, R. Azcón y A. Troncoso. 2009. Growth responses of micropropagated cassava clones as affected by Glomus Intraradices colonization. J. Plant Nutr. 32(2), 261-273. Doi: 10.1080/01904160802608601

Cuesta, I., E. Rengifo y M. Pérez. 2007. Influencia de diferentes dosis de Glomus mosseae sobre plántulas de $C$. odorata. En: Memorias $4^{\circ}$ Congreso Forestal de Cuba. La Habana, Cuba.

DANE (Departamento Administrativo Nacional de Estadística). 2004. Censo de producción de yuca para uso industrial. Separata de resultados. Bogotá, Colombia.

Falcón, E. 2010. Efecto de la aplicación de micorrizas arbusculares sobre la producción de posturas de Caoba del 
país (Swietenia mahagoni L. Jacq.) Homb. Cienc. Tecn. $14,1-11$

FAO. 2016. Cassava production statistics. En: Faostat, http://www.fao.org/faostat/es/\#data/QC; consulta: enero de 2018.

Filho, A.C. y M.A. Nogueira. 2007. Microbiota de solo e qualidade ambiental. Instituto Aronómico, Campinas, Brasil.

Grageda, O.A., A. Díaz, J.J Peña y J.A. Vera. 2012. Impacto de los biofertilizantes en la agricultura. Rev. Mex. Cienc. Agric. 3(6), 1261-1274.

Heberle, E.D.S., R.D.D. Armas, D.A. Heberle, S.L. Stürmer, L.A.M. Peruch, P.E. Lovato y C.R.F.S. Soares. 2015. Occurrence and structure of arbuscular mycorrhizal fungal communities in cassava after cultivation of cover crops as observed by the "PCR-DGGE" technique. Rev. Bras. Ciênc. Solo 39(5), 1292-1301. Doi: 10.1590/01000683rbcs20140216

Howeler, R. 2014. Sustainable soil and crop management of cassava in Asia: a reference manual. CIAT Publication No. 389. CIAT, Cali, Colombia.

João, J.P., A. Ruiz Martínez, L. Simó González y R. Rivera Espinosa. 2016. Efectividad de cepas de HMA en el cultivo de la yuca (Manihot esculenta Crantz) en dos tipos de suelos. Rev. Cult. Trop. 37(1), 48-56.

Lugtenberg, B. y F. Kamilova. 2009. Plant-growth-promoting rhizobacteria. Annu. Rev. Microbiol. 63, 541-556. Doi: 10.1146/annurev.micro.62.081307.162918

MADR (Ministerio de Agricultura y Desarrollo rural). 2003. Manejo social del campo 2002-2006. Bogotá, Colombia.

Molina, L., L. Mahecha y S. Medina. 2005. Importancia del manejo de hongos micorrizógenos en el establecimiento de árboles en sistemas silvopastoriles. Rev. Colomb. Cienc. Pecu. 18(2), 162-175.
Okon, I.E., M.G. Solomon y O. Osonubi. 2010. The effects of arbuscular mycorrhizal fungal inoculation and mulch of contrasting chemical composition on the yield of cassava under humid tropical conditions. Sci. World J. 10, 505-511. Doi: 10.1100/tsw.2010.59

Rienzo, J.A., F. Casanoves, M. Balzarini, L. González, M. Tablada y C.W. Robledo. 2015. Infostat. Universidad Nacional de Cordoba, Córdoba, Argentina.

Sánchez de Praguer, M. 1999. Endomicorrizas en agroecosistemas colombianos. Universidad Nacional de Colombia, Palmira, Colombia.

Séry, D.J.-M., Z.G.C. Kouadjo, B.R.R. Voko y A. Zézé. 2016. Selecting native arbuscular mycorrhizal fungi to promote cassava growth and increase yield under field conditions. Front. Microbiol. 7, 2063. Doi: 10.3389/ fmicb.2016.02063

Straker, C.J., A.J. Hilditch y M.E.C. Rey. 2010. Arbuscular mycorrhizal fungi associated with cassava (Manihot esculenta Crantz) in South Africa. S. Afr. J. Bot. 76(1), 102-111. Doi: 10.1016/j.sajb.2009.09.005

Suárez, L. y Mederos, V. 2011. Apuntes sobre el cultivo de la yuca (Manihot esculenta Crantz). Tendencias actuales. Cult. Trop. 32(3), 27-35. Doi: 10.3389/ fmicb.2016.02063

Sylvia D.M., J.J. Fuhrmann, P.G. Hartel y D.A. Zuberer. 1999. Principles and applications of soil microbiology. Ed. Prentice Hall Inc, Upper Saddle River, NJ, USA.

Vessey, J.K. 2003. Plant growth promoting rhizobacteria as biofertilizers. Plant Soil 255, 571-586. Doi: 10.1023/A:102603721689

Vila, O. 2012. Diagnóstico de la cadena de la yuca en Colombia. Generalidades y situación actual del cultivo de la yuca en Colombia. Ministerio de Agricultura y Desarrollo Rural (MADR), Bogotá, Colombia. 